\title{
Efeitos de um programa de exercícios físicos na flexibilidade em trabalhadores de escritório
}

\author{
Effects of a physical exercise program on flexibility \\ in office workers
}

\author{
Valquíria Aparecida de Lima*, Abdallah Achour Junior** \\ *Doutoranda em Saúde Coletiva pela Faculdade de Ciências Médicas da Unicamp (FCM-Unicamp), \\ **Universidade Estadual de Londrina
}

\section{Resumo}

Este estudo teve como objetivo avaliar os efeitos de um programa de exercícios no local de trabalho sobre a flexibilidade em trabalhadores de escritório e sua relação com a frequência semanal de participação. Quarenta e nove funcionários de escritório de uma empresa do setor farmacêutico, localizada na cidade de São Paulo, foram distribuídos randomicamente em três grupos de acordo com o número semanal de sessóes propostas: duas (RG2), três (RG3) e cinco (RG5) vezes por semana. O programa incluiu sessóes de exercícios de 10 minutos compostas por alongamento, resistência muscular localizada, massagem e técnicas de relaxamento, por um período de seis meses. Para mensurar a flexibilidade de punhos e coluna cervical foi utilizado um inclinômetro de dupla escala e o teste do Terceiro Dedo ao Chão para mensurar a flexibilidade da coluna lombar. Após a análise dos dados, verificou-se que houve aumento significante nos valores de flexibilidade de coluna cervical, coluna lombar e punhos $(\mathrm{p}<0,05)$ para todos os grupos randomizados. Este estudo demonstrou que a participação em um programa de exercícios no local de trabalho pode contribuir para o aumento da flexibilidade.

Palavras-chave: amplitude de movimento articular, ginástica laboral, exercícios de alongamento muscular, trabalhadores.

\begin{abstract}
This study was designed to evaluate the effects of a workplace exercise program on flexibility among office workers, and its relationship with the weekly frequency of participation. Forty nine office employees from a pharmaceutical company in the city of São Paulo were randomized into three groups according to the number of proposed weekly sessions: twice (RG2), three (RG3) and five (RG5) times a week. The program included ten-minute exercise sessions of stretching, resistance training, self-massage, massage and relaxation techniques for a period of six months. A dual-scale inclinometer was used to measure wrist and cervical spine flexibility and the fingertip-to-floor test was used to estimate the low back range of flexibility. Data demonstrated statistically significant improvement in flexibility of the cervical and lumbar spine and wrists $(\mathrm{p}<0.05)$ in all groups of randomization. This study demonstrated that participating in a workplace program of physical exercises might contribute to improve flexibility.
\end{abstract}

Key-word: range of motion, workplace exercises, muscle stretching exercises, workers.

Recebido em 24 de junho de 2014; aceito em 29 de junho de 2014.

Endereço para correspondência: Abdallah Achour Junior, Rua Antonio Pscchio, 200/2501, 86050-482

Londrina PR, E-mail: achour-junior@hotmail.com 


\section{Introdução}

Muitas empresas têm implementado programas de atividades físicas no local de trabalho como tentativa de reduzir a incidência e a severidade do desconforto musculoesquelético. Embora a efetividade desses programas ainda não tenha sido completamente comprovada, já que a maioria destes estudos tem sido conduzida com relativa deficiência metodológica [1,2], foram verificados efeitos benéficos do alongamento na prevenção de distúrbios musculoesqueléticos na coluna cervical [3], antebraço e punho [4], tais como síndrome do túnel do carpo, tendinite e tenossinovite, causados por ocupaçóes que requerem o uso frequente das máos em movimentos repetitivos $[5,6]$ e por, essa razão, o assunto tem merecido muita atenção $[7,8]$.

Estudos que mensurem os efeitos de programas de exercícios físicos realizados em situaçóes reais de trabalho, ainda são relativamente escassos. Portanto, o objetivo deste estudo foi verificar o efeito de um programa de exercícios físicos por um período de seis meses de duração, composto por resistência muscular, alongamento, massagem e relaxamento, oferecidos em séries de 10 minutos com diferentes frequências semanais, sobre indicadores de flexibilidade musculoesquelética de trabalhadores de escritório.

\section{Material e métodos}

Este trabalho de característica prospectiva incluindo intervenção e três momentos de coleta de dados: antes da intervenção, três e seis meses após início do programa - foi parte integrante das atividades do Centro de Promoçáo da Saúde (CPS) do Serviço de Clínica Geral do Hospital de Clínicas da Faculdade de Medicina da Universidade de São Paulo.

\section{Características da amostra}

Duzentos e vinte e oito funcionários de escritório de uma indústria farmacêutica localizada na cidade de São Paulo foram convidados a participar da pesquisa; 117 funcionários assinaram o Termo de Consentimento Livre e Esclarecido (TCLE) e 76 funcionários atenderam a pelo menos uma sessão de exercícios durante o período de duraçáo do programa. O estudo incluiu uma amostra de 49 dos 228 funcionários convidados $(21,5 \%), 44,9 \%$ do gênero masculino e $55,1 \%$ do gênero feminino, com média de idade de $32,1( \pm 10,3)$ anos e índice de massa corporal (IMC) de 22,7 $( \pm 2,1)$, que assinaram o TCLE, participaram da avaliação de flexibilidade e preencheram o inventário breve de dor durante o período do estudo.

Para organizar a frequência de participação no programa, os sujeitos foram alocados de forma randômica em três grupos, de acordo com o número proposto de sessóes semanais: duas vezes (RG2), três vezes (RG3) e cinco vezes por semana (RG5). Foram excluídos sujeitos que apresentaram quadros clínicos incompatíveis com o programa oferecido, como a presença de alguma incapacidade ou doença pré-estabelecida e sujeitos com necessidades físicas especiais.

A flexibilidade de coluna cervical, coluna lombar e punhos foram avaliados no início do programa (M0) e após três (M3) e seis meses (M6). Todas as avaliações ocorreram pela manhã, sem qualquer exercício prévio de aquecimento. $\mathrm{O}$ critério para a escolha da empresa foi a não participação de um programa de atividades físicas estruturado no ambiente de trabalho.

\section{Aspectos éticos}

O projeto foi previamente aprovado pela Comissão de Ética para Análises de Projetos de Pesquisa (CAPPesq) do Hospital das Clínicas da Faculdade de Medicina da Universidade de São Paulo.

Antes do início da pesquisa, todos os funcionários foram convidados, via sistema intranet de comunicação da empresa, a participar de uma palestra, na qual foram dadas explicaçóes sobre os objetivos e os procedimentos da pesquisa, sobre a aprovação do projeto de pesquisa pela CAPPesq do Hospital das Clínicas da Faculdade de Medicina da Universidade de São Paulo, e foi apresentada a pesquisadora. 


\section{Mensuraçáo da flexibilidade - inclinô- metro}

Para mensurar a flexibilidade da coluna cervical e punhos foi utilizado um inclinômetro mecânico. Seu mecanismo apresenta um dispositivo que ajusta em zero grau, a posição inicial do avaliado [9-13]. O procedimento para tomar as medidas foi extraído de Lea e Gerhardt [14].

\section{Mensuraçáo da flexibilidade de coluna lombar/isquiotibiais - teste do terceiro dedo ao cháo}

O teste do terceiro dedo ao chão, já descrito por outros autores [15-17], foi utilizado para mensurar em centímetros, com o auxílio de uma trena, a flexibilidade de coluna lombar e isquiotibiais.

Este teste foi escolhido por permitir aos trabalhadores uma maior percepção da sua flexibilidade, já que o teste pode ser realizado facilmente fazendo com que o examinado perceba seu estado inicial de flexibilidade.

Para efeito de cálculo foi considerada somente a maior das três medidas, tanto para os testes realizados com o inclinômetro quanto para o teste do terceiro dedo ao chão.

\section{Sessóes de exercícios}

O programa de exercícios foi composto por sessóes de exercícios que tiveram duração aproximada de dez minutos, com cinco minutos de intervalo para troca dos grupos, realizadas semanalmente no próprio local de trabalho, nas salas de reunióes disponibilizadas pela empresa, cinco dias por semana, das $9 \mathrm{~h}$ às $10 \mathrm{~h} 30$, resultando em aproximadamente seis sessóes por dia.

Para que não houvesse contaminaçáo da amostra, os participantes tiveram que se locomover para uma sala fechada onde as sessóes foram realizadas. Aproximadamente cinco ciclos de cinco semanas foram realizados, reiniciando a divisão acima após o final da $5^{\text {a }}$ semana, num total de 25 semanas de sessóes práticas, ministradas em dias úteis.

\section{Protocolo das sessóes de exercícios}

O conteúdo das sessões de exercícios previa abordar dois grupos musculares por semana, tendo como objetivo geral englobar, ao término de cada mês, as regióes corporais mais utilizadas nas tarefas diárias de trabalho.

Os exercícios foram divididos em exercícios de alongamento (60\%), técnicas de automassagem e relaxamento $(20 \%)$, e exercícios de resistência muscular localizada (20\%), com a seguinte divisão por regióes corporais: $1^{\mathrm{a}}$. semana: coluna lombar e isquiotibiais; $2^{\text {a }}$. semana: coluna cervical e ombros; $3^{a}$. semana: coluna torácica; $4^{a}$. semana: punhos e antebraço; $5^{\mathrm{a}}$. semana: coluna cervical, ombros e coluna lombar.

\section{Elaboração das sessóes de exercícios}

O tempo de permanência em cada exercício foi em média de 30 segundos, por ser um período de duração efetivo para aumento da flexibilidade [18]. Todas as aulas seguiram o seguinte protocolo para sua elaboração e aplicação: dois exercícios de alongamento específicos para cada grupo muscular avaliado, de acordo com o conteúdo definido para a semana e um exercício de relaxamento para o retorno ao estado de repouso pré-exercício para os grupos exercitados.

\section{Análise estatística}

O teste de Kolmogorov-Smirnov foi aplicado para todas as variáveis quantitativas com o objetivo de testar a normalidade de suas distribuições. Para todos os indicadores de flexibilidade, exceto para o teste do terceiro dedo ao chão, a distribuição pôde ser considerada normal.

Foi utilizada a ANOVA para comparação dos valores médios pré, 90 e 180 dias após a intervenção com o teste de Tukey para localizar as diferenças estatísticas nos vários momentos.

A análise estatística do teste de terceiro dedo ao chão foi feita por meio de testes não paramétricos de Friedman e o teste de Wilcoxon para localizar diferenças entre períodos.

Para todas as análises, o nível de significância adotado foi $\mathrm{p}<0,05$. O software Statistical 
Package for the Social Sciences (SPSS [versão 14.0]) foi empregado para os cálculos.

\section{Resultados}

As quantidades totais das sessóes de exercícios oferecidas ao longo do período de intervenção foram: Grupo RG2 - 39 sessōes; Grupo RG3 - 58 sessôes, Grupo RG5 - 97 sessôes. A participação semanal efetiva nas sessóes de exercícios pelos trabalhadores dos três grupos randomizados está ilustrada na Tabela I.

Comparaçôes utilizando ANOVA monofatorial e teste de Tukey indicaram diferenças estatisticamente significantes entre as médias de participação efetiva.

Ao estabelecer comparaçóes dos valores médios dos indicadores de flexibilidade entre os grupos randomizados no início da intervenção (pré-avaliação), não foi observada diferença estatisticamente significante em nenhum indicador. Assim, os grupos iniciaram o programa com níveis de flexibilidade estatisticamente semelhantes.

\section{Resultados da avaliação de flexibilidade}

Para o grupo randomizado para duas vezes por semana (RG2) (Tabela II), houve melhora significante da extensão de coluna cervical e de flexão e extensão de punhos após um período de apenas 90 dias de intervenção. Após 180 dias este grupo teve aumento significante $(\mathrm{p}<0,05)$ também para flexão de coluna cervical e terceiro dedo ao chão, em relação ao início do programa, além de outros incrementos em relação aos 90 dias. Os resultados sugerem que em 90 dias, com frequência de sessóes de duas vezes por semana, ocorre aumento da flexibilidade, mas que não é distribuído uniformemente. Com a continuidade do programa, algumas articulaçóes podem ter maiores aumentos de flexibilidade, o que evidencia a necessidade de se saber os valores mínimos de flexibilidade por articulaçáo que se deseja alcançar, para se propor 90 ou 180 dias como período apropriado de programa.

Para o grupo randomizado para três vezes por semana (RG3) (Tabela III), após 90 dias de intervenção houve diferença significante nos valores

Tabela I - Média e desvio padrão do número de sessóes efetivamente frequentadas por semana, de cada grupo randomizado $(R G)$ e comparação estatística.

\begin{tabular}{lcccc}
\hline \multicolumn{5}{c}{ Grupo Randomizado (RG) } \\
\hline & RG2 $(\mathbf{N}=18)$ & RG3 $(\mathbf{N}=15)$ & RG5 (N=16) & $P^{1}$ \\
\hline $\begin{array}{l}\text { Número de sessões } \\
\text { semanal }(X \pm D P)\end{array}$ & $1,39 \pm 0,50$ & $2,2 \pm 0,86$ & $3,13 \pm 1,10$ & 0,021 \\
\hline
\end{tabular}

Comparação por ANOVA monofatorial com teste de Tukey

Tabela II - Valores de média aritmética $(X)$ e desvio padrão $(D P)$ de flexibilidade com frequência de duas vezes semanal entre O (MO), 90 (M3) e 180 (M6) dias - Grupo RG2.

\begin{tabular}{lccc}
\hline \multicolumn{1}{c}{ Variáveis $(\mathbf{N}=18)$} & MO & M3 & M6 \\
\hline Flexão de Coluna Cervical & $\mathbf{X} \pm \mathrm{DP}$ & $\mathbf{X} \pm \mathrm{DP}$ & $\mathbf{X} \pm \mathrm{DP}$ \\
Extensão de Coluna Cervical & $71,9 \pm 8,9$ & $77,8 \pm 13,3$ & $83,1 \pm 11,4^{\circ}$ \\
Flexão de Punho Direito & $76,4 \pm 12,7$ & $90,6 \pm 15,8^{\circ}$ & $92,9 \pm 13,2^{\circ}$ \\
Extensão de Punho Direito & $40,3 \pm 8,7$ & $50,6 \pm 7,6^{\circ}$ & $55,6^{\circ} \pm 7,8^{\circ}$ \\
Flexão de Punho Esquerdo & $34,2 \pm 7,5$ & $45,6 \pm 10,6^{\circ}$ & $49,2 \pm 9,4^{\circ}$ \\
Extensão de Punho Esquerdo & $41,4 \pm 5,9$ & $50,0 \pm 13,3^{\circ}$ & $57,8 \pm 5,5^{\circ}$ \\
Terceiro Dedo ao Chão & $35,0 \pm 8,6$ & $45,0 \pm 8,4^{\circ}$ & $52,2 \pm 7,3^{\circ}$ \\
\hline
\end{tabular}

a- diferença estatística significante em relação a $M 0(p<0,05)$

$b$ - diferença estatística significante em relação a $M 3(p<0,05)$ 
médios de flexão e extensão de punho direito e flexão de punho esquerdo. Entretanto, após 180 dias não foi observada diferença significante entre os valores médios em comparaçáo com o início do programa apenas para extensão da coluna cervical.

O grupo randomizado para cinco vezes por semana (RG5) (Tabela IV), após 90 dias teve melhora significante de flexão cervical, flexão e extensão de punho direito e esquerdo. Após 180 dias foi observada diferença significante $(\mathrm{p}<0,05)$ entre os valores médios em comparação com o início do programa para todas as variáveis avaliadas.

\section{$\overline{\text { Discussão }}$}

O American College of Sports Medicine (ACSM) recomenda a realização de exercícios de alongamento com frequência mínima de três vezes por semana (frequência ideal entre 5 e 7 vezes) servindo-se de qualquer técnica de alongamento [19].
Neste estudo, os resultados relatados demonstram que o programa de exercícios no local de trabalho, com duração de 180 dias e duração de 10 minutos por sessão, contribuiu significativamente para o aumento da flexibilidade.

Estes resultados de desenvolvimento da flexibilidade após três e seis meses de intervenção foram estatisticamente significantes $(\mathrm{P}<0,05)$ em todos os grupos randomizados, lembrando que as médias e desvios-padrão de participação efetiva de cada grupo foram: $1,39 \pm 0,5$ no RG2, $2,2 \pm 0,86$ no RG3 e 3,13 $\pm 1,1$ no RG5.

Sainz de Baranda e Ayla [20] demonstraram aumento significante na flexão de quadril após 12 semanas de alongamento quando comparado com o grupo controle. Do mesmo modo, um programa de alongamento no local de trabalho entre funcionários de uma indústria farmacêutica [21] demonstrou um aumento estatisticamente significante de flexibilidade para todos os grupos musculares testados, após um período de alon-

Tabela III - Valores de média aritmética $(X)$ e desvio padrão $(D P)$ de flexibilidade com frequência de duas vezes semanal entre O (MO), 90 (M3) e 180 (M6) dias - Grupo RG3.

\begin{tabular}{lccc}
\hline \multicolumn{1}{c}{ Variáveis (N = 15) } & M0 & M3 & M6 \\
\cline { 2 - 4 } & $\mathbf{X} \pm$ DP & X \pm DP & X \pm DP \\
\hline Flexão de Coluna Cervical & $72,5 \pm 10,6$ & $81,3 \pm 10,7$ & $82,0 \pm 9,4^{\mathrm{a}}$ \\
Extensão de Coluna Cervical & $73,3 \pm 16,0$ & $84,3 \pm 15,3$ & $84,4 \pm 15,9$ \\
Flexão de Punho Direito & $31,1 \pm 8,6$ & $47,7 \pm 8,2^{a}$ & $55,3 \pm 9,9^{\mathrm{b}}$ \\
Extensão de Punho Direito & $32,0 \pm 6,8$ & $42,3 \pm 8,6^{\mathrm{a}}$ & $47,3 \pm 9,8^{\mathrm{b}}$ \\
Flexão de Punho Esquerdo & $34,6 \pm 8,6$ & $52,4 \pm 12,2^{a}$ & $53,3 \pm 9,7^{\mathrm{b}}$ \\
Extensão Punho Esquerdo & $30,9 \pm 7,4$ & $37,0 \pm 12,1$ & $50,3 \pm 9,7^{\mathrm{ab}}$ \\
Terceiro Dedo ao Chão & $11,2 \pm 8,3$ & $8,5 \pm 7,9$ & $2,8 \pm 4,3^{\mathrm{b}}$ \\
\hline
\end{tabular}

a- diferença estatística significante em relação a M0 $(\mathrm{p}<0,05)$

b- diferença estatística significante em relação a M3 $(\mathrm{p}<0,05)$

Tabela IV - Valores de média aritmética $(X)$ e desvio padrão $(D P)$ de flexibilidade com frequência de duas vezes semanal entre O (MO), 90 (M3) e 180 (M6) dias - Grupo RG5.

\begin{tabular}{lccc}
\hline & M0 & M3 & M6 \\
\cline { 2 - 4 } & $\mathbf{X} \pm \mathrm{DP}$ & $\mathrm{X} \pm \mathrm{DP}$ & $\mathrm{X} \pm \mathrm{DP}$ \\
\hline Flexão de Coluna Cervical & $66,2 \pm 9,0$ & $76,3 \pm 12,0^{\circ}$ & $84,4 \pm 11,5^{\circ}$ \\
Extensão de Coluna Cervical & $73,7 \pm 12,7$ & $82,2 \pm 19,7$ & $84,4 \pm 16,3^{\circ}$ \\
Flexão de Punho Direito & $33,8 \pm 6,8$ & $52,8 \pm 8,7^{\circ}$ & $57,2 \pm 9,7^{\circ}$ \\
Extensão de Punho Direito & $33,1 \pm 7,7$ & $46,6 \pm 7,9^{\circ}$ & $51,9 \pm 14,7^{\circ}$ \\
Flexão de Punho Esquerdo & $38,7 \pm 10,5$ & $55,7 \pm 9,6^{\circ}$ & $59,4 \pm 9,9^{\circ}$ \\
Extensão Punho Esquerdo & $30,9 \pm 8,1$ & $50,0 \pm 9,1^{\circ}$ & $56,9 \pm 11,9^{\circ}$ \\
Terceiro Dedo ao Chão & $13,3 \pm 12,8$ & $9,3 \pm 10,4$ & $2,9 \pm 3,2^{\circ}$ \\
\hline
\end{tabular}

a- diferença estatística significante em relação a $M 0(p<0,05)$ 
gamento de dois meses. Em outro programa de flexibilidade entre bombeiros municipais [16], também foi constatado que os sujeitos que participaram do programa por seis meses tornaram-se mais flexíveis que os não participantes.

Contudo, é muito difícil estabelecer comparaçôes com outras pesquisas, pois o nosso estudo não se delimitou apenas a realizar exercícios de alongamento; os de massagem e resistência localizada podem ter provocado variaçóes na flexibilidade. Além disso, as articulaçôes do punho e cervical são pouco pesquisadas se comparadas com as de coluna lombar e quadril.

No que diz respeito ao tempo de alongamento, um estudo demonstrou que, 15 segundos de alongamento para abdução de quadril foi tão efetivo quanto 120 segundos para o aumento da flexibilidade [22]. Em um experimento clínico [23], não foram encontradas diferenças entre 30, 60, 90 ou 120 segundos com alongamento estático na articulação do joelho. Desta maneira, o tempo em que se permanece em alongamento não parece ser um fator restritivo ao desenvolvimento da flexibilidade, pelo menos na resposta aguda e em programa com estudo transversal.

De interesse para facilitar a implementaçáo de programa de ginástica laboral será saber qual é o tempo mínimo necessário e frequência semanal para aumento da flexibilidade e correlacionar com a profilaxia de lesóes musculoesqueléticas, como já foi feito preliminarmente por Lima et al. [24].

Para que os resultados dos programas de ginástica laboral sejam bem avaliados é fundamental que sejam vistos, analisados e conduzidos como parte de um conjunto de ações multidisciplinares para a melhoria da saúde no ambiente de trabalho. Por isso, no período de intervençóes deste estudo, foram realizadas também palestras com o objetivo de estimular a diminuição do sedentarismo por meio da inserção de ginástica laboral no horário de trabalho, além de mostrar os benefícios para a qualidade de vida no trabalho e incentivar a adoção de pequenas práticas diárias de atividades físicas, como utilizar as escadas ao invés de elevadores e escadas rolantes. Com esse fato e diante da amplitude de tempo utilizado para o programa, é difícil propor somente exercícios de alongamento como forma preventiva de lesóes e com finalidade de identificar sua efetividade.
Estudos vêm sendo realizados para avaliar os efeitos dos exercícios de alongamento sobre a diminuição de dor e fadiga muscular relacionada ao trabalho $[25,26]$, em razáo de que a implantação de programas de exercícios no ambiente de trabalho é uma das açôes que estáo sendo utilizadas pelas empresas na tentativa de prevenir desordens musculoesqueléticas, cujos efeitos sobre fadiga, dores e lesōes ocupacionais têm despertado interesse da comunidade científica [27]. Contudo, vale ressaltar que é sempre importante destacar os valores de flexibilidade em associação com os exercícios de alongamento.

\section{Conclusão}

O estudo demonstrou que a participação em um programa estruturado de exercícios no local de trabalho ao longo de seis meses, com sessões de 10 minutos e diferentes frequências semanais pode contribuir para o aumento da flexibilidade em trabalhadores de escritório. Sugere-se, para estudos futuros, confirmar se a intervençáo estabelece um relacionamento causal entre desenvolvimento da flexibilidade e profilaxia de lesóes musculoesqueléticas.

\section{$\overline{\text { Referências }}$}

1. Costa BR, Vieira ER. Stretching to reduce workrelated musculoskeletal disorders: a systematic review. J Rehabil Med 2008;40:321-8.

2. Hess JA, Hecker S. Stretching at work for injury prevention: issues, evidence, and recommendations. Appl Occup Environ Hyg 2003;18(5):331-8.

3. Lin TY, Kaziyama HHS, Teixeira MJ, Simons DG. Síndrome dolorosa miofascial. In: Teixeira MJ, Lin TY, Kaziyama HHS, organizadores. Dor: síndrome dolorosa miofascial e dor músculo-esquelética. São Paulo: Rocca; 2006. p.105-18.

4. Couto HA. Ergonomia aplicada ao trabalho: conteúdo básico, guia prático. Belo Horizonte: Ergo; 2007.

5. Birkbeck MQ, Beer TC. Occupation in relation to the carpal tunnel syndrome. Rheumatol Rehabil 1975;14(4):218-21.

6. Luopajärvi T, Kuorinka I, Virolainen M, Holmberg M. Prevalence of tenosynovitis and other injuries of the upper extremities in repetitive work. Scand J Work Environ Health 1979;5(suppl 3):48-55. 
7. Janwantanakul P, Praneet Pensri P, Moolkay P, Jiamjarasrangsi WBMC. Development of a risk score for low back pain in office workers-a crosssectional study. BMC Musculoskelet Disord 2011;12(23):2-8.

8. Martins GC, Barreto SMG. Vivências de ginástica laboral e melhoria da qualidade de vida do trabalhador: resultados apresentados por funcionários administrativos do instituto de física da Universidade de São Paulo (Campus Sáo Carlos). Motriz Rev Educ Fís 2007;13(3):214-24.

9. Burton K. Measuring flexibility. Appl Ergon 1991;22(5):303-7.

10. Hole DE, Cook JM, Bolton JE. Reliability and concurrent validity of two instruments for measuring cervical range of motion: effects of age and gender. Man Ther 1995;1(1):36-42.

11. Hoving JL, Buchbinder R, Green S, Forbes A, Bellamy N, Brand C, et al. How reliably do rheumatologists measure shoulder movement? Ann Rheum Dis 2002;61(7):612-6.

12. Meirelles ES, Kitadai F. Aspectos clínicos e epidemiológicos da espondilite anquilosante. Rev Bras Reumatol 1999;39:30-4.

13. Viitanen JV, Kokko ML, Heikkilä S, Kautiainen $\mathrm{H}$. Neck mobility assessment in ankylosing spondylitis: a clinical study of nine measurements including new tape methods for cervical rotation and lateral flexion. $\mathrm{Br} \mathrm{J}$ Rheumatol 1998;37(4):377-81.

14. Lea RD, Gerhardt JJ. Range of motion measurements. J Bone Jt Sur Ser A Am 1995;77(5):784-98.

15. Gauvin MG, Riddle DL, Rothstein JM. Reliability of clinical measurements of forward bending using the modified fingertip-to-floor method. Phys Ther 1990;70(7):443-7.

16. Hilyer JC, Brown KC, Sirles AT, Peoples L. A flexibility intervention to reduce the incidence and severity of joint injuries among municipal firefighters. J Occup Med 1990;32(7):631-7.

17. Perret C, Poiraudeau S, Fermanian J, Colau MML, Benhamou MAM, Revel M. Validity, reliability, and responsiveness of the fingertip-to-floor test. Arch Phys Med Rehabil 2001;82(11):1566-70.
18. Ryan ED, Herda TJ, Costa P B, Defreitas JM, Beck TE, Stout J, Cramer J. Determining the minimum number of passive stretches necessary to alter musculotendinous stiffness. J Sports Sci 2009;27(9):957-61.

19. American College of Sport Medicine. ACSM's guidelines for exercise testing and prescription. 6th ed. Philadelphia: Lippincott Williams \& Wilkins; 2000.

20. Sainz de Baranda P, Ayala F. Chronic flexibility improvement after 12 week of stretching program utilizing the ACSM recommendations: hamstring flexibility. Int J Sports Med 2010;31(6):389-96.

21. Moore TM. A workplace stretching program. Physiologic and perception measurements before and after participation. AAOHN J 1998;46(12):563-8.

22. Madding SW, Wong JG, Hallum A, Medeiros JM. Effect of duration of passive stretch on hip abduction range of motion. J Orthop Sports Phys Ther 1987;8:409-16.

23. Ford GS, Mazzone MA, Taylor K. The effect of 4 different durations of static hamstring stretching on passive knee-extension range of motion. J Sport Rehabil 2005;14:95-107.

24. Lima VA, Aquilas AL, Ferreira Junior M. Efeitos de um programa de exercícios físicos no local de trabalho sobre a percepção de dor musculoesquelética em trabalhadores de escritório. Rev Bras Med Trab 2009;7:11-17.

25. Pereira CCD, López RFA, Vilarta R. Effects of physical activity programmes in the workplace (PAPW) on the perception and intensity of musculoskeletal pain experienced by garment workers. Work 2013;44:415-21.

26. Marangoni AH. Effects of intermittent stretching exercises at work on musculoskeletal in associated with the use of a personal computer and the influence of media on outcomes. Work 2010;36:27-37.

27. Choi SD, Woletz, T. Do stretching programs prevent work-related musculoskeletal disorders? Journal of SH\&E Research 2010;6(3). 